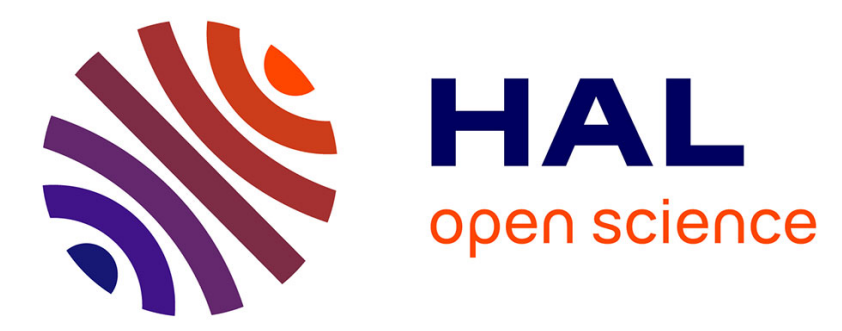

\title{
Broad working bandwidth and " endlessly" single-mode guidance within hybrid silicon photonics
}

\author{
K Bougot-Robin, J.-P Hugonin, M Besbes, Henri Benisty
}

\section{To cite this version:}

K Bougot-Robin, J.-P Hugonin, M Besbes, Henri Benisty. Broad working bandwidth and " endlessly " single-mode guidance within hybrid silicon photonics. Optics Letters, 2015, 40 (15), pp.3512-3515. 10.1364/OL.40.003512 . hal-01338291

\section{HAL Id: hal-01338291 \\ https://hal-iogs.archives-ouvertes.fr/hal-01338291}

Submitted on 28 Jun 2016

HAL is a multi-disciplinary open access archive for the deposit and dissemination of scientific research documents, whether they are published or not. The documents may come from teaching and research institutions in France or abroad, or from public or private research centers.
L'archive ouverte pluridisciplinaire HAL, est destinée au dépôt et à la diffusion de documents scientifiques de niveau recherche, publiés ou non, émanant des établissements d'enseignement et de recherche français ou étrangers, des laboratoires publics ou privés. 


\title{
Broad working bandwidth and "endlessly" single- mode guidance within hybrid silicon photonics
}

\author{
K. Bougot-Robin, ${ }^{1,2}$ J.-P. Hugonin, ${ }^{1}$ M. Besbes, ${ }^{1}$ And H. Benisty ${ }^{1, *}$ \\ ${ }^{1}$ Laboratoire Charles Fabry, Institut d'Optique Graduate School, Univ PSud, 2 Avenue Augustin Fresnel, F-91127 Palaiseau, France \\ ${ }^{2}$ Currently at Imperial College, Chemistry Department, Edel's Lab, SW7 2AZ, London, UK \\ ${ }^{*}$ Corresponding author: henri.benisty@institutoptique.fr
}

The successes of nonlinear photonics and hybrid silicon photonics with a growing variety of functional materials entail ever-enlarging bandwidths. It is best exemplified by parametric comb frequency generation. Such operation challenges the dielectric channel waveguide as the basis for guidance, because of the adverse advent of higher order modes at short wavelengths. Surprisingly, the popular mechanism of endlessly single-mode guidance [Opt. Lett. 22, 961 (1997).] operating in photonic crystal fibers has not been transposed within silicon photonics yet. We outline here the strategy and potential of this approach within planar and hybrid silicon photonics, whereby in-plane and vertical confinement are shown to be amenable to nearsingle-mode behavior in the typical silicon band, i.e., $\lambda=\mathbf{1 . 1} \boldsymbol{\mu \mathrm { m }}$ to $\sim \mathbf{5} \boldsymbol{\mu m}$. ( 2015 Optical Society of America

http://dx.doi.org/10.1364/OL.40.003512

The leveraging of nonlinear photonics and hybrid silicon photonics is well exemplified by comb-frequency generation through parametric $[\underline{1}, \underline{2}]$ or cascaded interactions [3]. These advances entail large frequency bandwidths, requiring clever dispersion management [4-7] on about two octaves $(\lambda=1.1 \mu \mathrm{m}$ to $\sim 5 \mu \mathrm{m})$, and can benefit from functional materials hybridized onto a silicon or silicon-on-insulator (SOI) platform, such as III-V semiconductors (see [] $]$ and references therein). Such broadband regimes challenge the dielectric channel waveguide as the basis for guidance: depending on the guide dimension, the risk arises of perturbing higher order modes at shorter wavelengths or deconfinement at longer ones.

We seek inspiration from the popular mechanism of "endlessly single-mode guidance" [9] of photonic crystal fiber (PCF), Fig. 1(a), to address this issue. The crucial point is that the cladding, carved in the same material as the core, is designed with periodic holes. Consequently, high-frequency modes see it as a higher effective index medium than low-frequency ones: the ability of these modes to squeeze their lobes repulsively off the low index inclusions (for PCF, air holes), raises the highest effective longitudinal-propagation constant of the cladding. For higher modes in a large core, Fig. 1(a), this suffices to provide leakage channels and maintain single-mode behavior "endlessly." This simple scheme did not find a genuine one-dimensional equivalent, in spite of clever early attempts [10]. There is a rich index map for integrated optics, whereby three refractive indices exist in any realistic channel/rib guidance (substrate, core, and cover). This blurs attempts to transpose the teaching of PCF whose cladding exploits only silica and air.

In this Letter, we focus on the SOI whose "index engineering" is mature [11]: we first show that the application to the SOI geometry of the "endlessly" single-mode guidance concept works with "endless" guidance on a broad range of about two octaves. We then argue that the addition of a hybrid stack based on III-V semiconductors can play most of the desired lightmatter interaction role, by relaxing the influence of vertical higher order modes if the layers are thick enough to accommodate the largest wavelengths of interest $(\sim 5 \mu \mathrm{m})$.

We analyze guidance in structures such as that of Fig. 1(b), and the hybrid one of Fig. 1(c). They are invariant along $z$, and

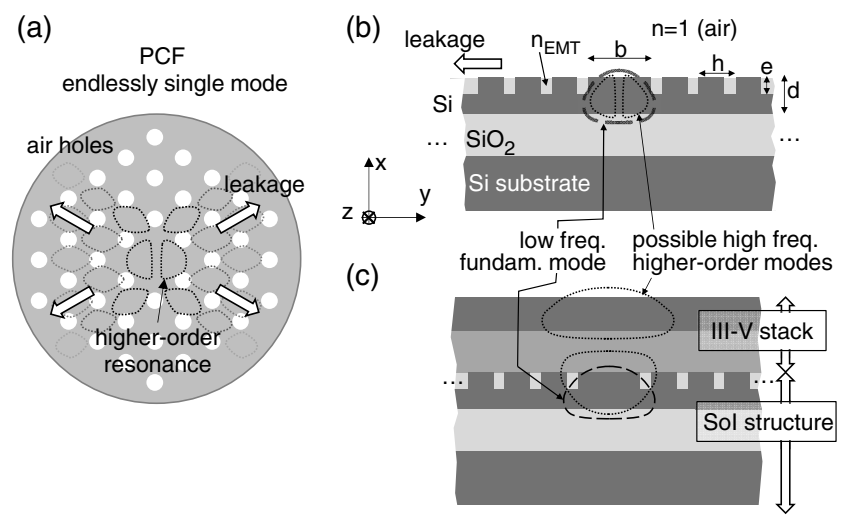

Fig. 1. (a) Photonic crystal fiber (PCF) and how a higher order resonance leaks into a high-effective-index cladding mode whose lobes are squeezed off air holes. (b) SOI structure with central ridge and laterally, periodic ridges. (c) SOI+III-V structure. 
are not directly related to photonic-crystal waveguides but are nevertheless the logical transposition of the fiber concept to an integrated-optics concept. That of Fig. 1 (b) has an index map $n(x, y)$, and classical Si thicknesses for operation around $1.5 \mu \mathrm{m}$. We use a variant of the rigorous coupled-wave analysis (RCWA) [12-19], whereby the finite vertical dimension of the system, $x$, is padded with perfectly matched layer (PML) mimicked by a complex-coordinate transform [12], being hence equivalent to infinite claddings. Technically, the use of this transform along $x$ with its reduction in computational domain size limits the need of higher Fourier terms (typically 35 terms here). We can also get the field lateral profile without an extended ydomain, thanks to Bloch boundary conditions [13]: the field Bloch parameters are known at the start of the periodic domain, and the profile can be obtained up to arbitrary $y$ in the periodic domain with the solution, even in the case of slow or absent decay. Numerically, the aperiodic Fourier modal method was benchmarked as very accurate in [18], see also [14]. Material dispersion is taken into account. We take the tabulated data of $\mathrm{Si}, n_{\mathrm{Si}}(\lambda)$ and silica $n_{\text {silica }}(\lambda)$ as in [4]. We study the resonant response to a point source $S$ to have a picture of guidance and leakage, following [16-18]. The system is pictured in Fig. 2(a). The periodic cladding of period $h$ determines leakage through its band structure. It can be studied as $\omega=\omega\left(K_{y}, \gamma\right)$, Fig. $2(\mathrm{~b})$, with $K_{y}$ the transverse vector, $\gamma$ the longitudinal wavevector along $z$, and $\omega$ the frequency (vacuum wavelength $\lambda_{0}$ ). At a given frequency and for a given mode branch, each interval $\left[\gamma_{\min }, \gamma_{\max }\right]$ reached at said frequency defines an allowed cladding band. Our goal is to maximize such bands to ensure leakage for all but a fundamental mode of the whole guide. The source $S$ of Fig. 2(a) is either of three electric and three magnetic dipoles along the three axes, addressing any mode symmetry. Compared to [19], we rather impose the frequency $\omega$ and scan $\gamma$ to find the scattering matrix poles. This matrix is derived from the source through the core part to each side of the periodic cladding. Precisely, by calculating the field's imaginary part $\mid \operatorname{Im}[$ field $(S)] \mid$ at the source location $S$, we get profiles of resonances versus $\gamma$ such as Fig. 2(c) for the six source terms (in log scale), and by the same token, information on the bands as discussed later.

We start with a 50/50 duty cycle and a cladding period $h=0.6 \mu \mathrm{m}$, fairly larger than a typical half-wavelength at $\lambda_{0}=1.5 \mu \mathrm{m}$ in $\mathrm{Si}$ or also in planar SOI with typical $n_{\text {eff }}=2.8: \lambda_{0} / 2 n_{\text {eff }}=0.268 \mu \mathrm{m}$. The silicon thickness is here $d=500 \mathrm{~nm}$, etched by $e=250 \mathrm{~nm}$ groove. The defect size is $b=1.2 \mu \mathrm{m}$, large enough to otherwise accommodate a quasiTE mode up to large wavelengths $(\lambda=4.5 \mu \mathrm{m})$ without too much deconfinement. In Fig. 2(a), we fill the grooves with a composite air-silicon material of index $n_{\mathrm{EMT} d}(\lambda)$, EMT referring to "effective material theory" $[8,20-22]$, and $d$ to "dispersive": a broad range of indices can be made by nano-pattering silicon at modestly sub-wavelengths scales. We refer for simplicity to these dispersive media by the index $n_{\mathrm{EMT}}$ for $n_{\mathrm{Si}}=$ 3.5 exactly $\left[3.5=n_{\mathrm{Si}}(1.343 \mu \mathrm{m})\right]$, i.e., we use $n_{\mathrm{EMT} d}(\lambda)=$ $1+\left(n_{\mathrm{EMT}}-1\right)\left(n_{\mathrm{Si}}(\lambda)-1\right) /(3.5-1)$ with fixed $n_{\mathrm{EMT}}$. Note that a dispersionless EMT material gives a very similar behavior and a fully dispersionless stack still quite so. Conversely, an accurate modeling is nevertheless relevant for nonlinear comb generation $[\underline{1}-\underline{3}]$.
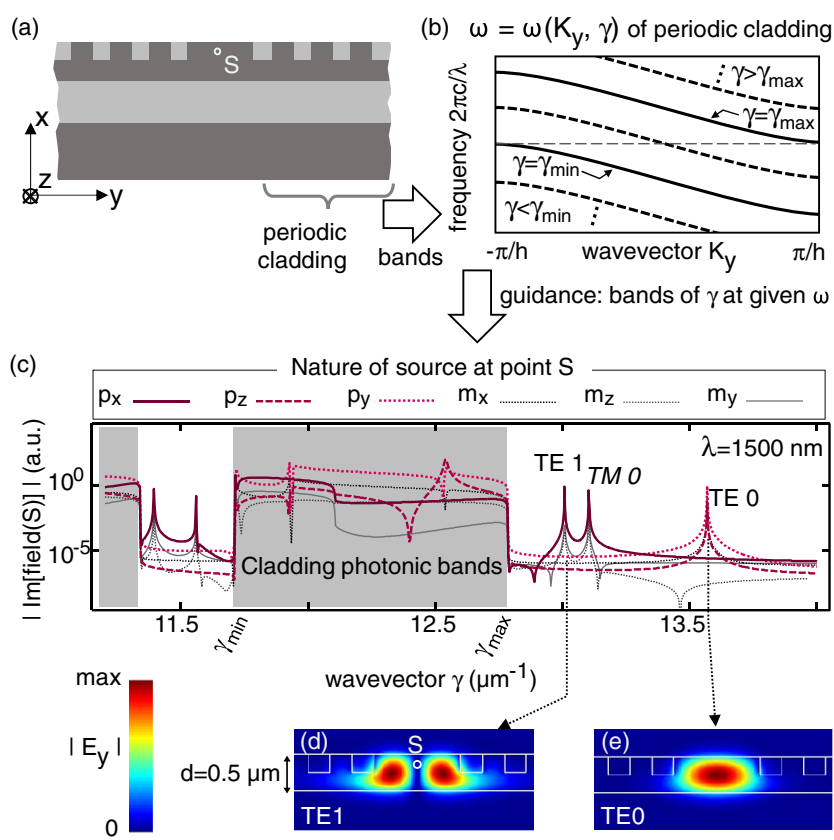

Fig. 2. System studied, with (a) source $S$ and periodic cladding; (b) generic cladding band structure of a given mode $\omega=\omega\left(K_{y}, \gamma\right)$ in first Brillouin zone $K_{y}$, parametrized by $\gamma$ : at a given frequency, an allowed $\gamma$ band arises in an interval $\left[\gamma_{\min }, \gamma_{\max }\right]$. (c)(e) $\lambda=1.5 \mu \mathrm{m}$ study of the basic structure with $n_{\mathrm{EMT}}=1.5$ and 50/50 duty-cycle. (c) Modal behavior, seen through the imaginary field response $\mid \operatorname{Im}[$ field $(S)] \mid$ at point $S$ to the six kinds of source terms. The gray areas with always high response are photonic bands. The area of low response are forbidden bands with localized modes. (d) TE1 mode profile, $E_{y}$ component; (e) TE0 mode profile, same. Note in (d) that $S$ stands at a zero of $E_{y}$, hence the distinct source responses versus TE0.

Figure 2(c) holds all we need: we checked that the claddingallowed bands tightly correspond to gray areas of Fig. 2(c), with higher response to the source. In forbidden bands, except discrete mode poles, the response is low as the energy leaving the system vanishes. Thus, truly guided modes occur at peaks within the white areas signaling forbidden bands. Concerning our quest of "endlessly" single mode guidance, while the TE0 mode shown in Fig. 2(e) fits in the defect, we see in Fig. 2(c) how the limited extent of allowed bands leaves enough room for our large defect to support a second TE1 guided mode, shown in Fig. 2(d). We now want to extend allowed bands till such short-wavelength TE1 modes merge in them.

In Fig. $\underline{3(\mathrm{a})}$, we study at $\lambda_{0}=1.5 \mu \mathrm{m}$ a $25 / 75$ duty cycle with still $n_{\mathrm{EMT}}=1.5$. We, hence, extend the allowed band up to $\gamma=13.1 \mu \mathrm{m}^{-1}$ instead of $12.75 \mu \mathrm{m}^{-1}$ in Fig. 2(c), thus, up to a higher effective index $n_{\text {eff }}=\gamma \lambda_{0} / 2 \pi$. Correspondingly, only a TE0 mode lies in the upper forbidden area, the TE1-like peak being repelled into the continuum at $\gamma<13.1 \mu \mathrm{m}^{-1}$. The TE0 mode profile in Fig. 3(c) fully resembles that of Fig. 2(e): as expected, a relatively large mode area provides this insensitivity.

An unbalanced duty cycle is not the only way to weaken periodicity: a lower index contrast also does. We thus try a rather high EMT index $n_{\mathrm{EMT}}=2.9$ keeping a 50/50 duty cycle, in 
Fig. 3(b). The allowed band now reaches nearly $\gamma=$ $13.25 \mu \mathrm{m}^{-1}$. Besides the single mode in this area, the TE2 mode now also has a very narrow window between allowed bands. Together with the large offset in $\gamma$, this makes unlikely the coupling to TE2, so we effectively approach a single-mode operation.

Checking how much room is left for suppressing TE1 is the issue addressed by Fig. $3(\mathrm{~d})$. For the $n_{\mathrm{EMT}}=$ 2.9 [b] cladding of Fig. $3(\mathrm{~b})$, we are at the vertical dashed line. By pushing up the frequency to $\lambda_{0}=1.42 \mu \mathrm{m}$, we facilitate squeezing the TE1 mode in the large defect. As shown in Figs. $3(\mathrm{e})-3(\mathrm{f})$, we skirt the single-mode limit there: the TE1 mode is extremely deconfined, while the TE0 mode is as confined as in Fig. 2(e). By performing a similar study at several $n_{\mathrm{EMT}}$ values from 2.8 to 3.4 , we outline the domain of TE single-mode operation in Fig. 3(d).

We now visualize what the TE0 mode looks like at large wavelengths where a single-mode behavior is natural, exploring mode profiles up to $\lambda_{0}=4.5-5 \mu \mathrm{m}$ in a hope to cover 1.5 octaves. Beyond $\sim 5 \mu \mathrm{m}$, the silica absorption onset of SOI is detrimental. In Fig. 4, we depict the TE0 mode for the same configurations (a), (b) as seen in Fig. 3. The mode has swollen similarly in both cases (we normalized the color map to silicon's strongest field to ease comparison). There is more confinement

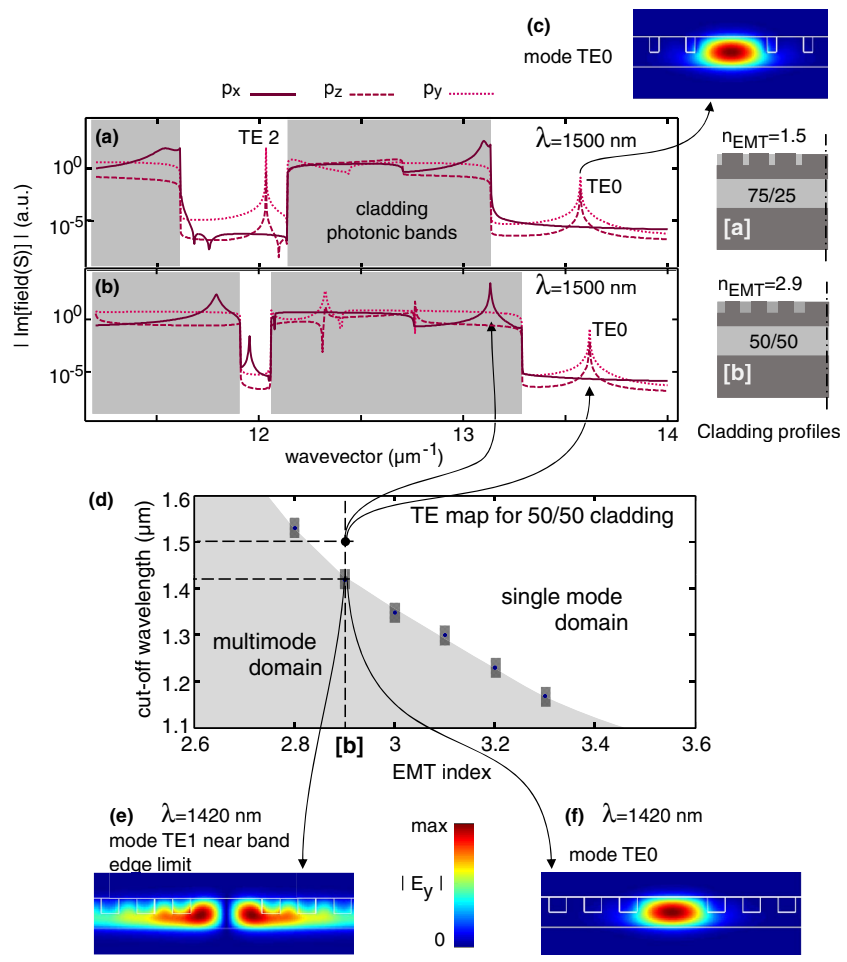

Fig. 3. (a) Modal study with only electrical response shown for $1.5 \mu \mathrm{m}$ and claddings with $75 / 25$ duty cycle and $n_{\mathrm{EMT}}=1.5$; (b) same for $50 / 50$ duty cycle and smaller contrast, $n_{\mathrm{EMT}}=2.9$; insets [a,b] on the right side show sketches of these cladding; (c) mode TE0 in the [a] cladding case looks identical with TE0 of more confined structures; (d) TE1 cut-off wavelength of structures with variable $n_{\mathrm{EMT}}$, the value $n_{\mathrm{EMT}}=2.9$ of [b] cladding is indicated; (e), (f) cut-off situation at $1.42 \mu \mathrm{m}$, the second-mode TE1 is just above cut-off (profile with slow decay). This TE1 mode would thus leak in a cladding mode at $1.5 \mu \mathrm{m}$, as desired, but would remain guided at $\lambda<1.42 \mu \mathrm{m}$. The TE0 mode remains well confined. in (a) (cladding[a]) as could be expected by the larger forbidden band. We are thus reaching a vertical deconfinement regime, not so surprisingly since the silicon slab with $d=500 \mathrm{~nm}=$ $\lambda_{0} / 9$ is below the typical $\lambda_{0} / 2 n=650 \mathrm{~nm}$ limit.

By a spectral quest of the fundamental mode, we can draw the phase dispersion of the TE0 mode, $n_{\text {eff }}=\gamma \lambda / 2 \pi$, Fig. $\underline{4(\mathrm{c})}$, as a function of wavelength (upper axis) and frequency $\nu=c / \lambda$ in $\mathrm{THz}$ (lower axis). Of great interest for comb and parametric generation is the group index $n_{\text {eff }}+\nu\left(d n_{\text {eff }} / d \nu\right)$, it is relatively stable around 3.5-3.9 over the two octaves.

We can now wonder whether we can compensate also for the vertical swelling (as well as manage dispersion) by getting closer to the original fiber endlessly single-mode concept: to this end we cannot afford a thicker silicon (a vertical TE1 mode would appear), but we can attempt to surround the central core by "allowed modes" in the $z$-direction as well, not only in the SOI xy plane. This is similar to the low-index "horizontal slot" of [ $[-7]$, albeit with less index contrast: this option is made possible in the moderate index contrast regime by the advent of hybrid oxide-free InP-on-Si silicon photonics, whereby bonding of a III-V heterostructure (InP-based) to nanostructured silicon has been demonstrated $[20,21]$.

The stack we explore is that of Fig. $5(\mathrm{~b})$, with a thicker silicon slab $(700 \mathrm{~nm})$ with $50 / 50$ duty cycle grooves identical in shape to those of $[\mathrm{b}]$ above. It consists of an extra 400-nm-thick InP layer (index $n \approx 3.17$ [20]), with a 300-nm top-quaternary layer (GaInAsP alloy with bandgap wavelength $<1.42 \mu \mathrm{m}$ ) having the same index as $\mathrm{Si}$ for simplicity. No patterning is made in these layers. To compensate the expected TE0 profile "swelling" toward the III-V and maintain in-plane confinement, a lower $n_{\mathrm{EMT}}=2.6$ instead of 2.9 is adopted.

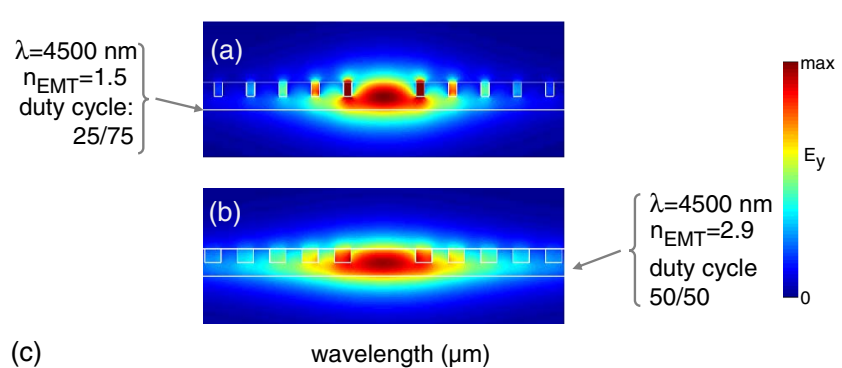

(c)

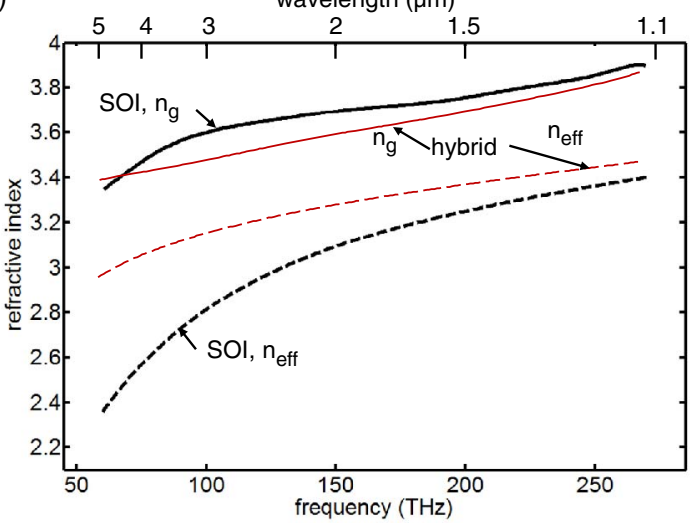

Fig. 4. (a), (b) Modes at $4.5 \mu \mathrm{m}$ for the [a,b] cladding of Fig. 3 are given in (a), (b), respectively. Note the pronounced field extrema within the EMT in (a). (c) Phase and group index dispersions of SOI (thick line) and hybrid (thin line) structures. 
Figure 5(a) reminds the mode profile of the stand-alone SOI stack, whereas Figs. 5(c) $-5(\mathrm{e})$ show the TE0 mode profile at $4.5,2.5$, and $1.5 \mu \mathrm{m}$. The avoidance of deconfinement is most visible on the silica side below silicon with a weaker tail there (for these maps, the coordinate-transform $x$ boundaries are set beyond the map limits, while they are closer for extensive scans). And overall, there is a better overlap of the TE0 profiles at different frequencies. Both factors are positive toward enhanced nonlinear mid-infrared operation. As for the higher-order mode patterns, Figs. 5(f) $-5(\mathrm{i})$ show a selection at the three same wavelengths as above, hence the rows [(c) and (f); (d), (g), and (h); (e) and (i)]. The occurrence of a "TE1 vertical mode profile" is clear [Figs. 5(f), 5(g), 5(i)]. But (1) its profile nests in the top 300-nm layer even up to $4.5 \mu \mathrm{m}$ [Fig. 5(f)], and (2) it propagates laterally (allowed bands) except at short wavelength [Fig. 5(i)], but it is quite deconfined versus TE0. As for the apparently localized "vertical TE2 mode," Fig. 5(h), we checked that it leaks as well. Finally, we did a scan for this structure and got the effective indices of Fig. 4(d) (thin lines). We note a weaker phase-index dispersion and more regular group-index dispersion, with very weak second-order terms.

In this Letter, we do not explore the full parametric study that would map, for instance, the exact onset loci of higher order modes in the rich parameter space, as this obviously depends on application and technology-related choices. We believe that the more important point is to expose an embodiment strategy for the "endlessly" (broad working range) singlemode-guidance principle, first in a single transverse direction, (a) $\mathrm{SOI}+$ air

$4.5 \mu \mathrm{m}$

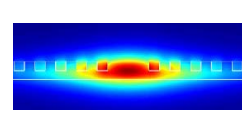

$4.5 \mu \mathrm{m}$

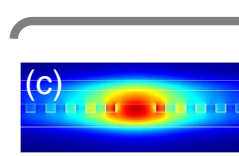

$2.5 \mu \mathrm{m}$

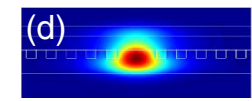

$1.5 \mu \mathrm{m}$

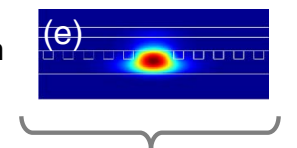

TEO

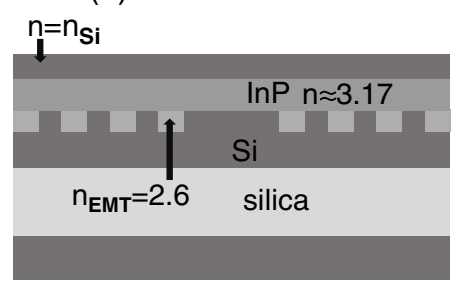

(b) $\mathrm{SOI}+\mathrm{III}-\mathrm{V}$

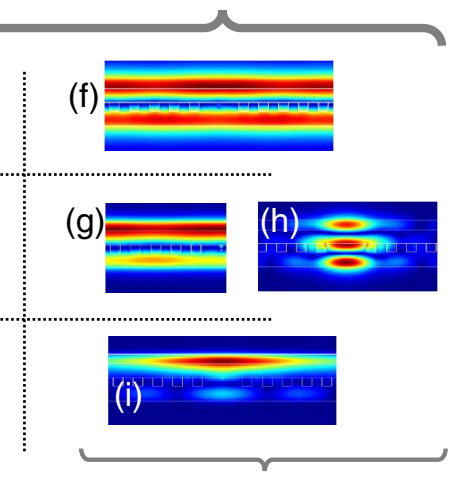

vertical high-order modes
Fig. 5. Various modes and their $y$ component. (a) Reference mode at $4.5 \mu \mathrm{m}$ in SOI stack; (b) sketch of III-V on SOI; (c)-(e) mode in SOI+III-V stack at 4.5, 2.5 and $1.5 \mu \mathrm{m}$; (f), (g) the second "vertical 1" mode at 4.5 or $2.5 \mu \mathrm{m}$ tends to peak in the high-index layer at shorter wavelengths, but has no in-plane confinement; (h) third vertical mode; (i) top-split lobe of the TE high-order mode at $1.5 \mu \mathrm{m}$. but also in the "vertical" direction, similarly to ongoing efforts [5-7]. Even if our strategy provides a mitigation rather than a total cut-off of the higher-order modes, it would have a clear interest given the large sensitivity of nonlinear processes to overlap integrals underlying light-matter interactions.

To conclude, we have successfully exported to the "silicon realm" the strategy of "endlessly" single-mode guidance developed in PCF. We adapted the clever principle of these PCF studies concerning the shaping of large "escape windows" in the cladding. In terms of spectral width, a broad working range was obtained, from $\sim 5 \mu \mathrm{m}$ the onset of silica absorption, to $\sim 1.15 \mu \mathrm{m}$ the silicon bandgap. With the rest of the photonic toolbox, planar-based channel guide geometries can challenge current fiber functions. Our strategy holds for nonlinear hybrid as well as standard silicon photonics.

Funding. French Agence Nationale de la Recherche (L'Agence Nationale de la Recherche) (COHEDIO).

\section{REFERENCES}

1. B. Kuyken, T. Ideguchi, S. Holzner, M. Yan, T. W. Hänsch, J. Van Campenhout, P. Verheyen, S. Coen, F. Leo, R. Baets, G. Roelkens, and N. Picqué, Nat. Commun. 6, 6310 (2015).

2. A. G. Griffith, R. K. Lau, J. Cardenas, Y. Okawachi, A. Mohanty, C. Poitras, A. L. Gaeta, and M. Lipson, Nat. Commun. 6, 6299 (2015).

3. A. Hugi, G. Villares, S. Blaser, H. Liu, and J. Faist, Nature 492, 229 (2012).

4. H. Benisty and N. Piskunov, Appl. Phys. Lett. 102, 151107 (2013).

5. L. Zhang, Y. Yue, R. G. Beausoleil, and A. E. Willner, Opt. Express 19, 8102 (2011).

6. L. Zhang, J. Mu, V. Singh, A. M. Agarwal, L. C. Kimerling, and J. Michel, J. Sel. Top. Quantum Electron. 20, 136 (2014).

7. L. Zhang, C. Bao, V. Singh, J. Mu, C. Yang, A. M. Agarwal, L. C. Kimerling, and J. Michel, Opt. Lett. 38, 5122 (2013).

8. A. Talneau, C. Roblin, A. Itawi, O. Mauguin, L. Largeau, G. Beaudouin, I. Sagnes, G. Patriarche, C. Pang, and H. Benisty, Appl. Phys. Lett. 102, 212101 (2013).

9. T. A. Birks, J. C. Knight, and P. S. J. Russell, Opt. Lett. 22, 961 (1997).

10. E. Silvestre, P. S. J. Russel, T. A. Birks, and J. C. Knight, J. Opt. Soc. Am. A 15, 3067 (1998).

11. J. H. Schmid, P. Cheben, P. J. Bock, R. Halir, J. Lapointe, S. Janz, A Delâge, A. Densmore, J. Fedeli, T. J. Hall, B. Lamontagne, R. Ma, I. Molina-Fernandez, and D.-X. Xu, IEEE Photon. J. 3, 597 (2011).

12. J. P. Hugonin and P. Lalanne, J. Opt. Soc. Am. A 22, 1844 (2005).

13. Q. Cao, P. Lalanne, and J.-P. Hugonin, J. Opt. Soc. Am. A 19, 335 (2002).

14. G. Colas des Francs, J.-P. Hugonin, and J. Ctyroky, Opt. Quantum Electron. 42, 557 (2011).

15. E. Silberstein, P. Lalanne, J.-P. Hugonin, and Q. Cao, J. Opt. Soc. Am. A 18, 2865 (2001).

16. G. Lecamp, J.-P. Hugonin, and P. Lalanne, Opt. Express 15, 11042 (2007).

17. G. Lecamp, P. Lalanne, and J.-P. Hugonin, Phys. Rev. Lett. 99, 023902 (2007).

18. P. Bienstman, S. Selleri, L. Rosa, H. P. Uranus, W. C. L. Hopman, R Costa, A. Melloni, L. C. Andreani, J. P. Hugonin, P. Lalanne, D. Pinto, S. S. A. Obayya, M. Dems, and K. Panajotov, Opt. Quantum Electron. 38, 731 (2007).

19. Q. Bai, M. Perrin, C. Sauvan, J. P. Hugonin, and P. Lalanne, Opt. Express 21, 27371 (2013).

20. K. Bougot-Robin, A. Talneau, and H. Benisty, Opt. Express 22, 23333 (2014).

21. K. Bougot-Robin, C. Pang, X. Pommarede, A. Itawi, A. Talneau, J.-P. Hugonin, and H. Benisty, IEEE J. Lightwave Technol. 32, 3283 (2014).

22. C. Pang and H. Benisty, Photon. Nanostr. Fundam. Appl. 11, 145 (2013). 